\title{
Intonational processing is incremental and holistic
}

Timo B. Roettger ${ }^{1,2}$, Daniel Turner² \& Jennifer Cole²

1 Universitetet i Oslo

2 Northwestern University

Please cite as: Roettger, T. B., Turner, D., \& Cole, J. (2020, October 14). Intonational processing is incremental and holistic. https://doi.org/10.31234/osf.io/nhbgs

Corresponding author:

Timo B. Roettger

timo.b.roettger@gmail.com

Authorship contribution (following CRediT: https://casrai.org/credit/):

TBR: Conceptualization, Methodology, Data Curation, Formal Analysis, Investigation, Project administration, Writing - Original Draft, Writing - Review \& Editing, Visualization, Supervision

DT: Conceptualization, Methodology, Data Curation, Formal Analysis, Investigation, Writing Review \& Editing, Visualization, Project administration

JC: Conceptualization, Methodology, Resources, Project administration, Writing - Review \& Editing, Supervision, Funding acquisition 


\title{
Intonational processing is incremental and holistic
}

\begin{abstract}
Speakers modulate the intonation of an utterance to express essential communicative functions, and while intonational pitch contours span entire utterances, intonational melodies can be characterized as a sequence of discrete tonal events. A tonal event may constrain the interpretation of a temporally distant tonal event, and the entire tonal sequence is potentially relevant for recognizing a speaker's communicative intention. The question arises whether listeners process intonational information as soon as they become available (incremental processing) or whether they wait until they have access to the entire intonation contour with all its tonal events (holistic processing). In a visual world eyetracking experiment, we explored how and when American English listeners integrate a sequence of two pitch accents relative to the discourse status of different referents. Analyses of listeners' fixation patterns suggest that listeners incrementally process pitch accents as soon as they appear in the signal, and use this information to reduce uncertainty about the referents of both local and downstream expressions. Listeners also process early and late pitch accents in relation to one another, such that early cues in the utterance can restrict later inferences and late cues can be used to resolve uncertainty associated with earlier cues. These findings have implications for models of intonational processing, for which neither a local processing strategy nor a holistic view alone are sufficient. Effective comprehension of intonational events requires maintaining perceptual information long enough to integrate it with downstream intonational events. Open data, scripts, and materials can be retrieved here: https://osf.io/ 2wecs/.
\end{abstract}

Keywords: prosody, intonation, sentence comprehension, visual world paradigm 


\section{Introduction}

Speech is a complex physical signal that humans interpret with apparent ease, to understand not only the propositional meaning of an utterance, but also the speaker's intentions and mental state. This information is distributed over the utterance in many structurally distinct acoustic events, for instance, through sequences of phones and syllables that comprise words, or through a sequence of pitch accents and phrasal edge-marking tones that comprise intonational patterns. Some of these acoustic events are critical for signaling propositional content, while others serve primarily as cues to pragmatic information. The challenge for the listener is to know how to differentially attend to these acoustic events and to update their interpretation of utterance meaning in relation to acoustic cues as they come online.

This paper focuses on the role of intonation in listeners' interpretation of utterance meaning. In many languages, including English, speakers modulate fundamental frequency (corresponding to what we perceive as pitch) to signal non-propositional meaning related to the speaker's pragmatic intentions (i.e., speech acts), their social stance or their emotional state (e.g. Bolinger 1989, Bänziger \& Scherer 2005, Cruttenden 1986, Cutler 1997, Gussenhoven 2004, Ladd 2008, Dahan 2015, Holiday 2016). We henceforth refer to utterance-wide pitch modulation expressing nonpropositional meaning as 'intonation'. Depending on the type of intonational event and its location within the utterance, these events can signal very different meanings (Pierrehumbert \& Hirschberg 1990).

The psycholinguistic literature offers several accounts of how listeners process intonation when comprehending language. One view holds that intonation is incrementally processed, i.e. information is integrated as soon as it becomes available. Evidence for this view comes from studies showing that listeners rapidly integrate intonational cues to interpret discourse-pragmatic meaning (Dahan et al. 2002, Weber et al. 2006, Ito \& Speer 2006, Kurumada et al. 2014, Roettger \& Franke 2019). For example, when looking at a scene with a blue cup, a red cup, and a blue bottle and hearing instructions to take "the blue..." with a rising pitch movement on "blue", English and German listeners respond immediately with looks to the blue cup, 
rather than the blue bottle. This response reflects the listeners' knowledge of the intonational conventions of their language (English or German), where rising pitch movements are frequently associated with referents that semantically contrast with a discourse-salient alternative (blue cup - red cup). In addition to these behavioral results, inappropriate and thus unexpected intonation have been shown to immediately elicit neurophysiological responses corresponding to prediction error (e.g. Heim \& Alter 2006, Baumann \& Schumacher 2012). This process of incremental interpretation is in line with linguistic processing models, such as the chunk-and-pass model by Christiansen and Chater (2016), which assume that constraints on perceptual memory force comprehenders to process perceptual data immediately, compressing detailed information into abstract representations of linguistic categories for further processing.

The 'now-or-never' account of the chunk-and-pass processing model is, however, difficult to reconcile with a key property of prosody, including intonation, and that is that the acoustic cues to prosody, i.e., the rhythmic and melodic aspects of speech including fundamental frequency, are perceptually evaluated relative to other prosodic cues in the same phrase (Swerts \& Geluykens 1994, Krahmer \& Swerts 2001, Katz \& Selkirk 2011, see Cole 2015 for an overview). For instance, the pitch level of a word is perceived as high or low relative to the pitch events in its surrounding environment. Moreover, downstream pitch events can affect the interpretation of earlier pitch events (Dennison 2010, Dennison \& Schafer 2010). Such findings suggest an alternative model of holistic processing, where listeners wait until the end of the utterance, or at least the end of an intonational phrase, to compile acoustic information and relate prosodic cues to one another, interpreting intonational meaning for the utterance (or intonational phrase) as a whole.

While the literature cited above has been influential in advancing processing models of intonation, empirical support for those models is limited. Most studies investigate only a single intonational event, commonly the right-most pitch accent in an intonational phrase, termed the 'nuclear accent'. In linguistic models of intonation, the nuclear accent expresses pragmatic meaning related to focus and the information status of referring expressions (Halliday 1967, Cruttenden 1986). Yet it is common for utterances to have more complex intonation patterns that include prenuclear pitch accents in earlier parts of the utterance. The relevance of prenuclear pitch ac- 
cents for utterance meaning remains unclear. Some evidence indicates that prenuclear accents in English are variable in production (Chodroff \& Cole 2019); optionally placed for rhythmic purposes only (Büring 2007, Calhoun 2010); with lesser acoustic and perceptual prominence than nuclear accents (Cole et al. 2019); and disregarded by listeners when learning new form-meaning associations (Kapatsinski 2017). Collectively, these findings suggest a lesser role for prenuclear accents in conveying linguistic meaning. Yet, other studies have found that prenuclear pitch accents affect listeners' judgments about the status of upcoming referents as focused or discoursegiven (Gussenhoven 1983, Breen et al. 2010, Birch \& Clifton 1995, Bishop 2017; Petrone \& D'Imperio 2011, Petrone \& Niebuhr 2014, Braun \& Asano 2013). And at least one study on German (Braun \& Biezma 2019) shows that a prenuclear accent can influence the interpretation of the prenuclear-accented word itself; a rising-falling prenuclear pitch accent can activate semantic alternatives, indicating a contrastive interpretation of the prenuclear accented word. This latter body of evidence suggests that incoming pitch accents are incrementally and locally interpreted.

This paper presents results from a visual world eye tracking experiment that investigates how American English listeners process intonational events occurring early and late in an utterance for interpreting referential meaning. The findings support the incremental account, showing that listeners immediately integrate and interpret information about a pitch accent occurring early in an utterance, but they also reveal a limited type of backward inference, where downstream accents influence the referential interpretation of earlier expressions, as predicted in the holistic processing account.

\section{Method}

This study was preregistered on the 30th of September 2019 at https://osf.io/wkf6x/.

\subsection{Participants}

We tested 39 speakers of American English, recruited from the undergraduate Linguistics Subject Pool at Northwestern University. Following preregistered protocol, we excluded non-native American English speakers, early multilinguals, and participants with self-reported learning, attention or hearing disability $(n=5)$. 


\subsection{Procedure}

Participants were seated in front a 27 -inch computer screen (2560x1440 resolution) and an eye tracking device (SR-Research Eyelink system sampling with $1000 \mathrm{~Hz}$ ). Participants saw a visual scene with four pictures displayed in the corners of the screen (see Fig. 1c). Pictures displayed one of four different animals (Fig. 1B) dreaming about one of eight different food objects (Fig. 1A). Participants had to click on the picture corresponding to an auditory instruction identifying one of the animals dreaming about a particular type of food. Participants were told that they would hear a speaker that has word naming problems, i.e. the speaker frequently forgets some of the names and substitutes them with the filler word "thingy", which we take to be semantically empty in these contexts. In these situations, participants were instructed to guess the intended picture, in spite of the missing noun, without explicit instruction how to make this inference.

A

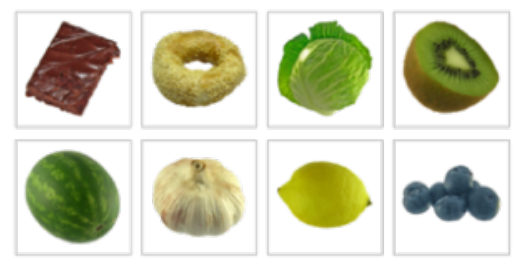

B

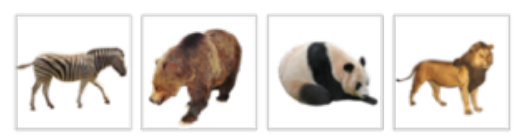

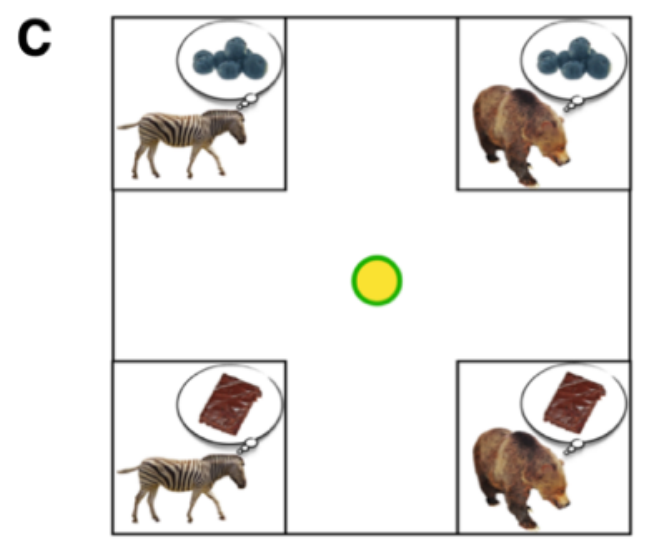

Figure 1: A - Object pictures; B - Animal Pictures; C - Example screen.

Participants were first presented with 16 trials in a familiarization phase, followed by 96 test trials. On test trials, participants clicked a start button to initiate audio playback of a trigger sentence (1).

(1) Trigger sentence: Click on the zebra that dreams about the berries.

On each trial, the displayed pictures were systematically related to the trigger sentence, such that there was a picture that displayed the same scene as the trigger 
sentence (here zebra + berries), one showing a different animal dreaming about the same object (here grizzly + berries), one showing the same animal and a different object (zebra + brownie), and one showing a different animal and a different object (grizzly + brownie). Below we refer to the animal or food object that differs from the one mentioned in the trigger sentence as the "contrastive" referent. The participant clicked on the picture matching the trigger sentence, after which the pictures disappeared and, after $1000 \mathrm{~ms}$, reappeared in the same locations, at which time the participant heard one of three test sentences, illustrated in (2).

(2) Test sentences:

(a) Now, click on the thingy that dreams about the berries instead. [Contrastive; Given]

(b) Now, click on the thingy that dreams about the brownie instead. [Given; Contrastive]

(c) Now, click on the thingy that dreams about the berries again. [Given; Given]

Crucially, the test sentence contained a semantically empty place holder for the first referent ("thingy") allowing us to investigate the integration of prosodic information without lexical guidance. Test sentences differed in their intonation patterns (see below) and their respective discourse relationship to the first instruction. (2a) refers to a contrastive subject and the same object given in the trigger sentence (1), (here, grizzly + berries). (2b) refers to the given subject and contrastive object (here, zebra + brownie). (2c) refers to the given subject and given object. After selecting a picture, participants received visual feedback (colored frame around the target picture). 


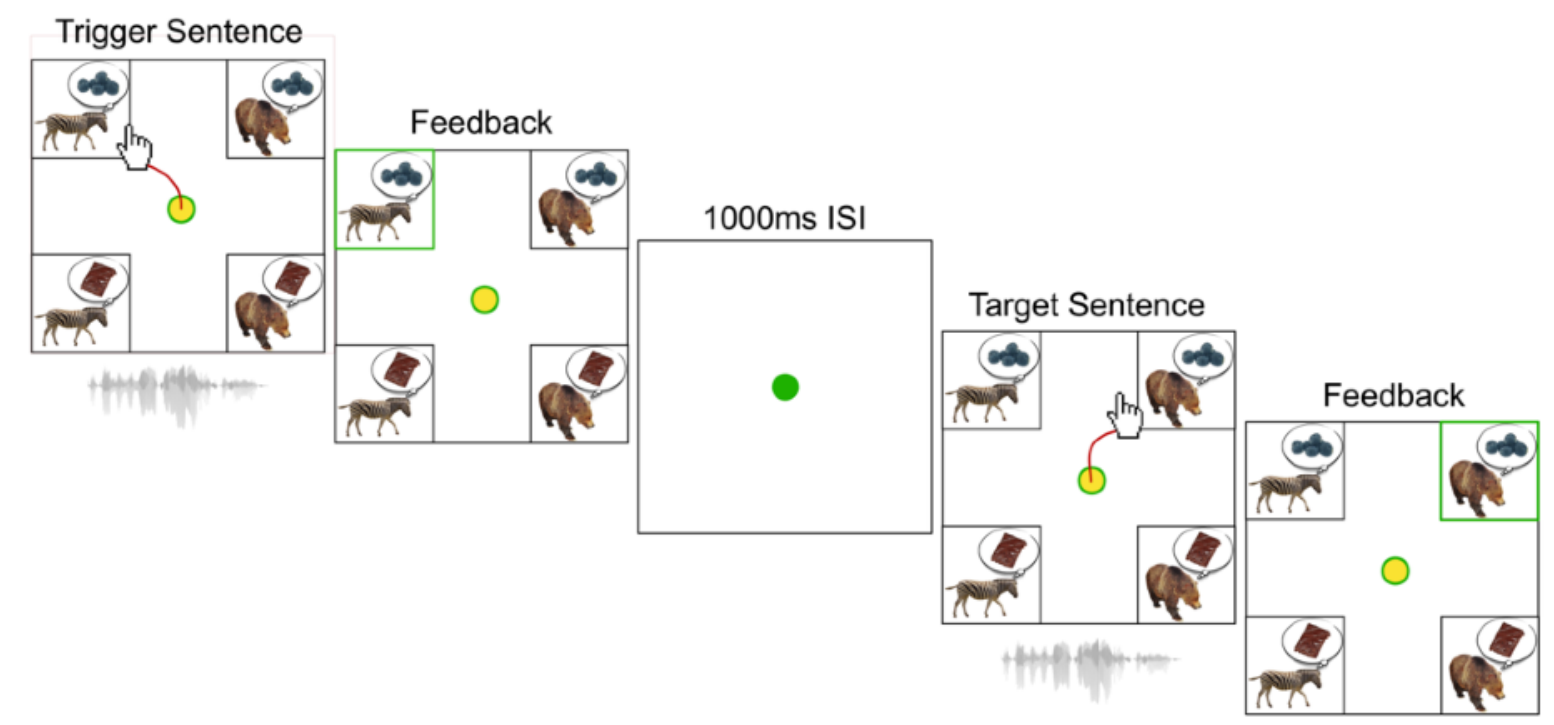

Figure 2: Trial structure. When hearing the trigger sentence, listeners had to click on the correct image (here: zebra + berries). After visual feedback and a 1000ms Inter-Stimulus-Intervall, listeners heard the target sentence.

\subsection{Materials}

The visual stimuli are from the BOSS corpus (Brodeur et al., 2010, see stimuli here: osf.io/4fp5s). Animals and objects were combined resulting in 32 distinct target pictures. Each target picture was used in association with each prosodic condition, resulting in 96 test trials.

Acoustic stimuli were recorded by a trained speaker of American English (female) (see details in preregistration: osf.io/wkf6x/). (2a) was realized with a rising-falling pitch accent on thingy and an unaccented object NP (henceforth "NP1 accented"). (2b) was realized with accent on the object NP and thingy unaccented ("NP2 accented"). (2c) was realized with accents on both thingy and the object NP ("Both NPs accented"). For the target sentences, we selected productions best suited for use as resynthesis baselines for both the first part of the utterance ("Now click on the thingy that..."), the second part of the utterance ("...dreams about the OBJECT") and the adverb ("again/instead"). We then resynthesized the pitch contour of the pitch accents of interest in order to ensure that the pitch accent on thingy (in 2a) has the same pitch excursion as the pitch accent on the object NP (in 2b). Resynthesis was done with the PSOLA function in Praat (Boersma \& Weenink, 2017) (see preregistra- 
tion for details), followed by cross-splicing the first part of the utterance (always the same within condition) with the second part of the utterance (containing the $2^{\text {nd }} \mathrm{NP}$ ) and the adverb, respectively. Example pitch tracks from the stimuli are displayed in Fig. 3 and stimuli audio files can be accessed here: osf.io/4fp5s.

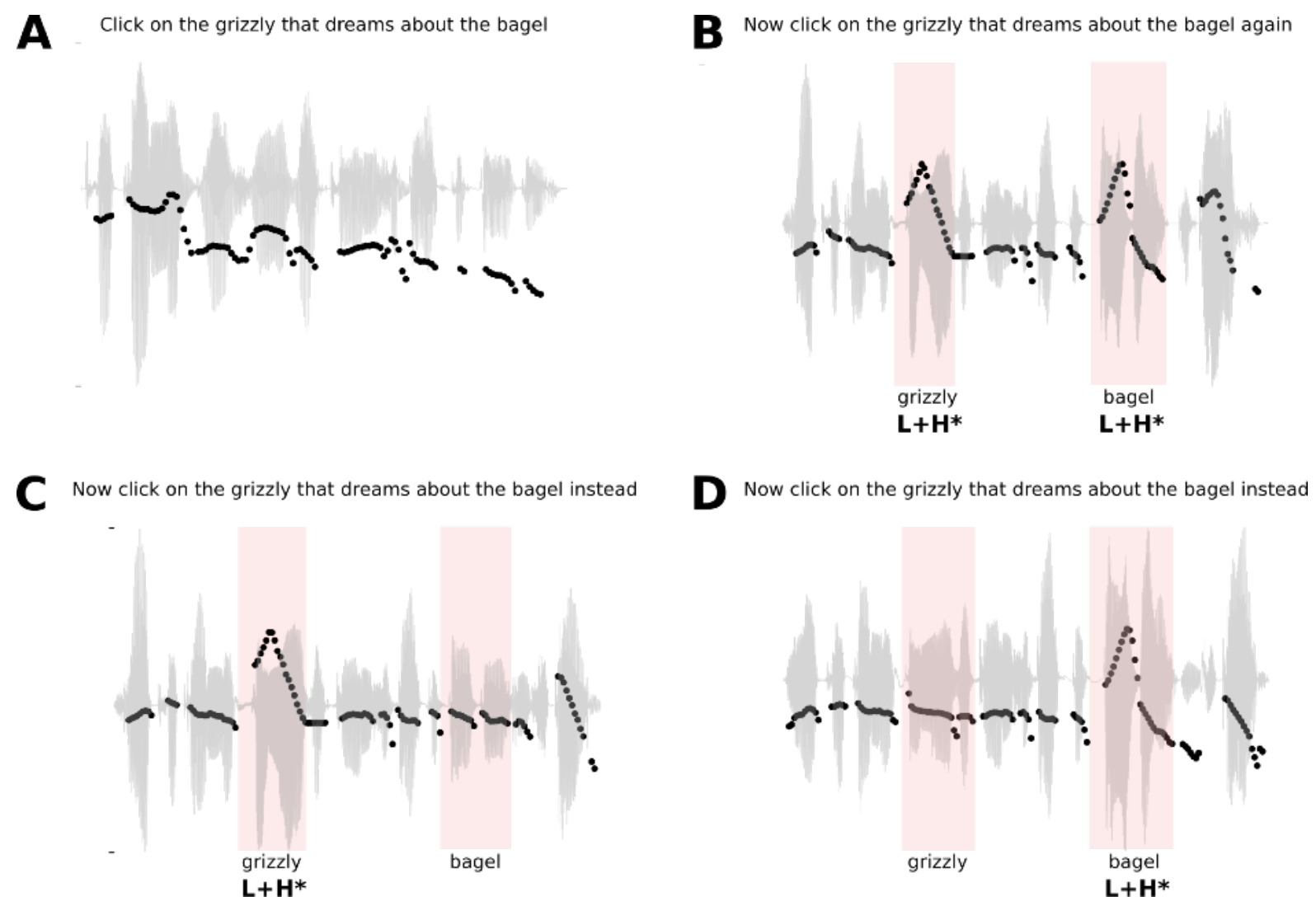

Figure 3: Example intonation contours (fundamental frequency range $50-450 \mathrm{~Hz}$ ). A - trigger sentence in condition $\mathrm{A}$ (mostly flat with $\mathrm{H}^{*}$ on sentences subject and object); $\mathrm{B}-$ contour with $\mathrm{L}+\mathrm{H}^{*}$ on both sentence subject and object paired with a given interpretation. $\mathrm{C}$ - contrastive subject contour with $\mathrm{L}+\mathrm{H}^{*}$ on subject; $\mathrm{D}-$ contrastive object contour with $\mathrm{L}+\mathrm{H}^{*}$ on object;

\subsection{Data processing}

We recorded participants' decisions (clicks) for trigger sentences (see (1), serving as catch trials) and test sentences (2). Participants' gaze fixations were recorded using a SR-Research EyeLink 1000+ eye tracking system. We defined four relevant temporal windows of the utterances: From the start of the utterance up to "thingy" (early window), from the onset of "thingy" up to onset of the object NP (1st NP window), from the onset of the object NP up until the onset of the adverb (2nd NP window), and 
from the onset of the adverb until the response was been made (late window). The onset of any landmark was defined by its acoustic onset plus a time lag of $200 \mathrm{~ms}$, the commonly assumed time lag for initiating a saccade (Allopenna et al. 1998). For each trial, fixation durations were aggregated over time windows for each response alternative. The resulting proportions were then converted in two binary preference measures: A NP1-preference index (the animals) and an NP2-preference index (the objects), based on whether the proportion of fixations to the target referent (animal/ object) was larger than the competitor referent, or smaller. For example, on trials where the target referent was the given animal, the competitor referent was the contrastive referent, and vice versa. This preference operationalization is conservative, as we do not have any specific hypotheses regarding the magnitude and/or temporal development of gaze preferences within the specified time windows.

\subsection{Statistical Analysis}

We fitted Bayesian hierarchical logistic regression models to both NP1 and NP2 preferences as a function of dummy-coded factors: TIME window (early, $1^{\text {st }} \mathrm{NP}, 2^{\text {nd }}$ NP, and late), PROSODY (NP1 accented, NP2 accented, Both NPs accented), scaled TRIAL numbers as well as their three-way interaction, using the package brms (Buerkner 2016) in R (R Core Team, 2017). ${ }^{1}$ Models include random intercepts for participant and target referent, and by-participant and by-referent random slopes for the predictors and their interactions. We specified weakly informative priors for all relevant coefficients (see https://osf.io/s3grp/). For all relevant means and differences between them $(\delta)$, we report the expected mean values under the posterior distribution and their $95 \%$ credible intervals (Crls). If a hypothesis states that $\delta>0$, we judge there to be compelling evidence that the hypothesis is supported if zero is not included in the $95 \% \mathrm{Crl}$ of delta. Data tables and scripts can be accessed here: osf.io/4fp5s.

\subsection{Predictions}

For each prosodic condition, there are three important comparisons to consider. We are interested in how listeners' fixation preferences for the referent of the 1 st NP

\footnotetext{
1 We refrain from detailing the intricate three-way interactions involving the trial numbers. Most effects remain inconclusive with only a few exceptions, indicating rather weak trial effects across the experiment. See Materials for additional information.
} 
change from the early to the NP1 time windows (i) indicating a potential local integration of prenuclear accent information. We are also interested in preference changes from the NP1 to NP2 time windows (ii), indicating how the accumulation of prenuclear and nuclear accent information affects referential interpretation of NP1. Finally, we are interested in how fixation preferences for the referent of the $2^{\text {nd }}$ NP change from the early to the NP1 time windows (iii), indicating a potential anticipation of the NP2 referent based on the prenuclear information.

If listeners incrementally process intonation, pitch accents will be immediately locally interpreted. In that case, we expect an increase in fixation preference to the contrastive subject when hearing an early accent in both 'NP1 accented' and 'both NPs accented' conditions.

If listeners holistically process intonation, i.e. wait until the end of the utterance to compile and interpret, reference assignment should wait at least until NP2 is heard. We expect no change in looks triggered by accent information on NP1, but once NP2 becomes available, listeners are expected to infer the referential interpretation of NP1. Thus, we expect an increase in looks to the target NP1 as late as the NP2 window.

\section{Results}

Looking at the fixation patterns across time (see Fig. 4), it becomes clear that listeners are biased to look at the referents introduced by the (preceding) trigger sentence. The picture with the given animal and object, represented by the green line (both given), starts higher than expected by chance and the blue line (both contrastive) starts lower. This initial bias is, however, quickly modulated by the incoming speech information. As predicted, listeners use intonational information in the prenuclear region to guide their fixation preferences early on. 


\section{Fixation proportions across time}

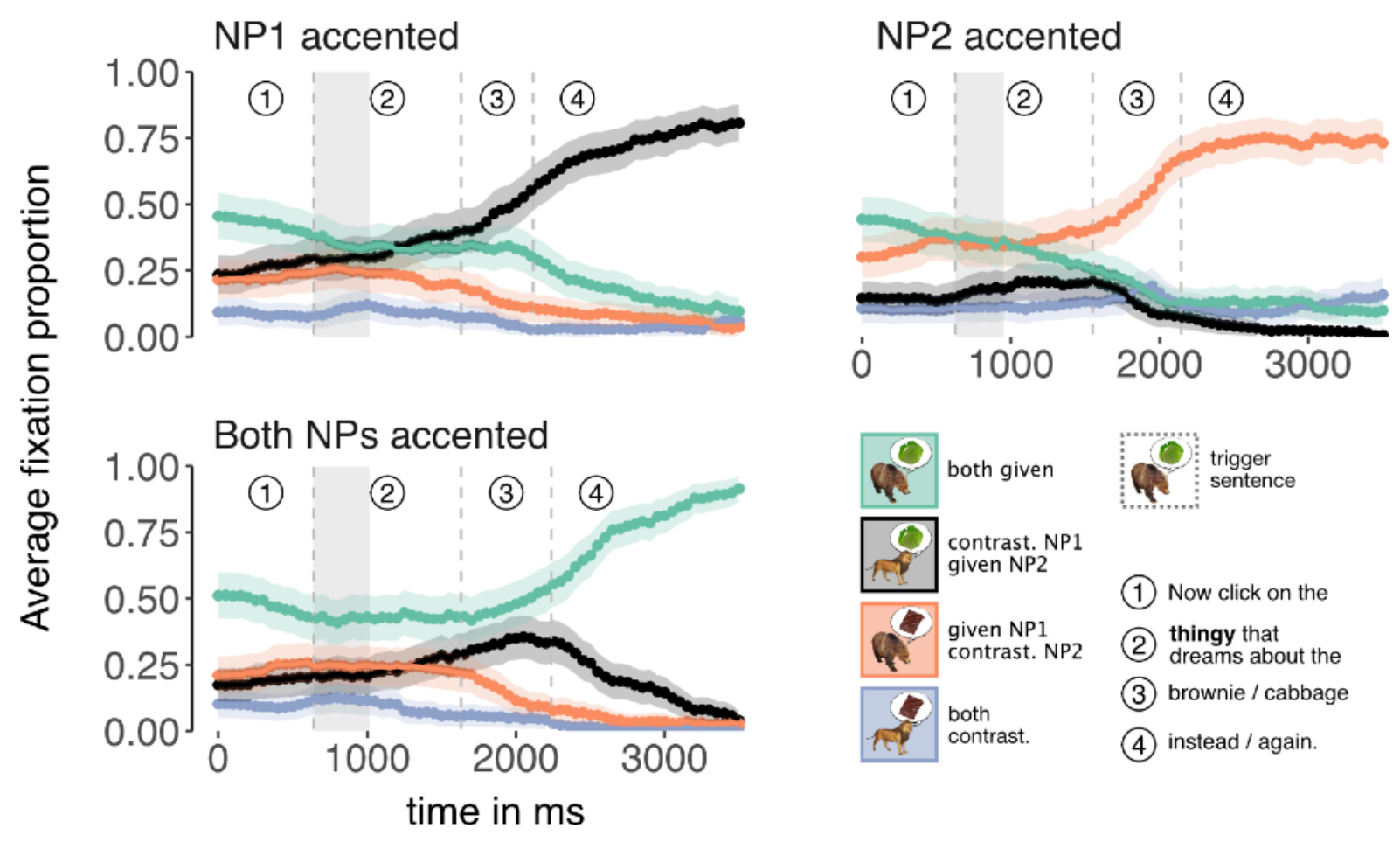

Figure 4: Mean fixation proportions across conditions (panels), picture types (color) and time (xaxis). Error margins corresponds to the standard error calculated by SD / (Vnumber of participants). Dashed lines correspond to the acoustic landmarks delimitating the time windows early (1), NP1 (2), NP2 (3), and late (4). The shaded area indicates the position of NP1 providing relevant prenuclear information.

\subsection{Looks to the first referent}

Fig. 5 displays the aggregated fixation preferences for the animals (= 1st NP, first row) and the objects ( $=2 \mathrm{nd} \mathrm{NP}$, second row) across three time windows: early, $1^{\text {st }}$ $\mathrm{NP}$, and $2^{\text {nd }} \mathrm{NP}$ (as described above). We find compelling evidence for three effects: Listeners locally interpret an early pitch accent as evidence for a contrastive NP1 (transition (a) in Fig. 4); this interpretation becomes strengthened on hearing an unaccented NP2 (transition (b)); and listeners use the absence of an early accent as evidence for a contrastive referent for NP2 (transition (c)). 


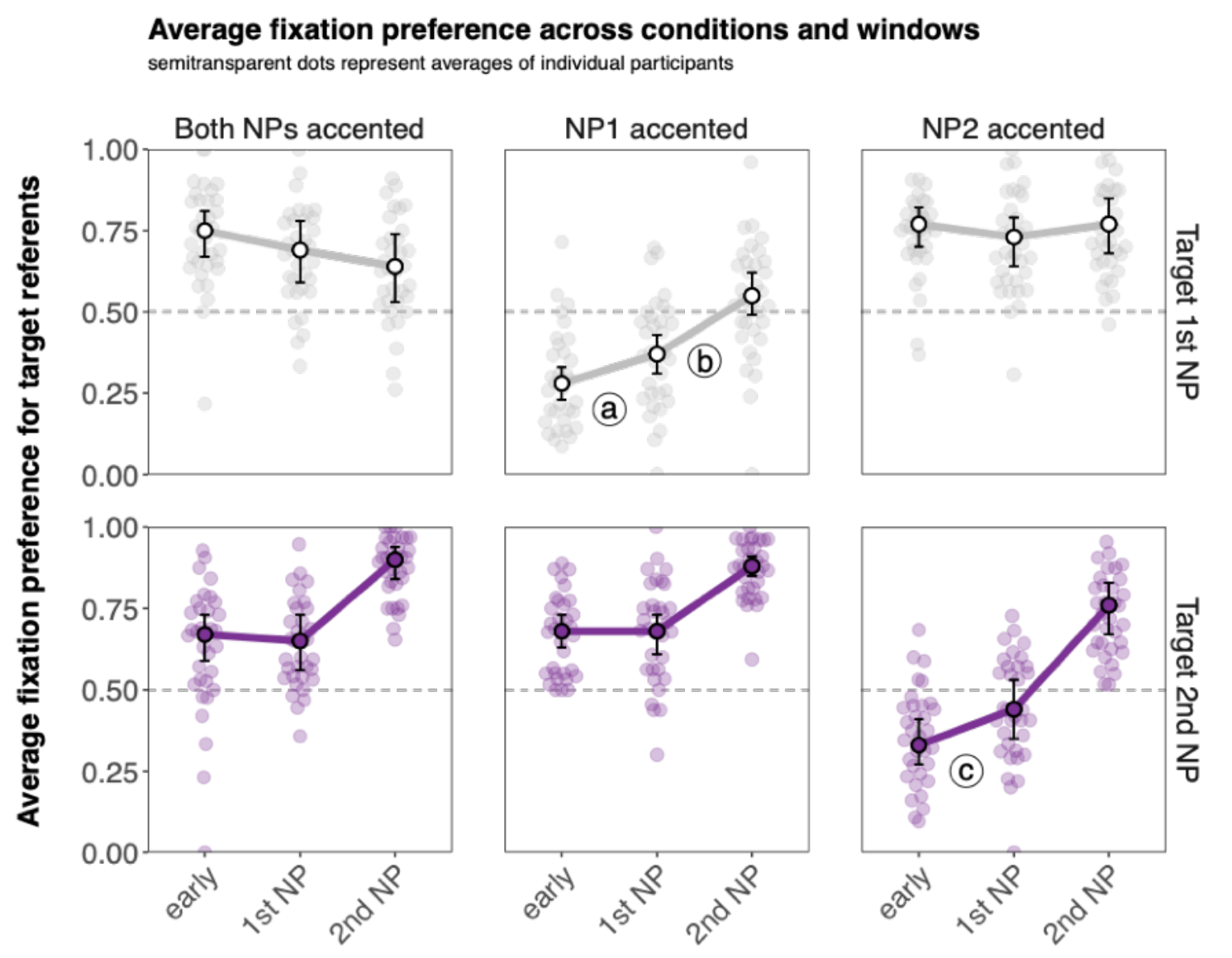

Figure 5: Aggregated fixation preferences to target NP across conditions (columns), referents (rows), and time windows (x-axis). Solid points and whiskers correspond to posterior means and $95 \% \mathrm{Crls}$ based on the models described above. Semitransparent points correspond to participant averages.

Looking at fixation preferences to NP1 (top row, Fig. 5), there is compelling evidence that listeners attend to prenuclear accent status in the 'NP1 accented' condition (left panel). There is an increase in looks to the target referent from the early window $(28 \%)$ to the NP1 window $(37 \%)$. The difference of the posterior distributions is compelling ( $\left.\beta_{\text {logodds }}=-0.44,95 \% \mathrm{Crl}=[-0.66,-0.21]\right)$. Hearing an accent on NP1 leads to substantially more looks to a referent that contrasts with the previously mentioned referent. In other words, the pitch accent is immediately locally interpreted (as contrastive). The same pattern can also be numerically observed for intonation contours with two accents (right panel). Listeners are more likely to look to the contrastive competitor (which in this case is not the target referent), when hearing an accent on thingy: There is a decrease in looks to the target referent from the early window $(75 \%)$ to the NP1 window (69\%). However, this condition shows substantially 
more variability, leading to less certain estimates of this difference $\left(\beta_{\text {logodds }}=0.28\right.$, $95 \% \mathrm{Crl}=[-0.07,0.64])$. We could not identify a systematic source of this variability. We, thus, assume it is unsystematic sampling noise. Between these two early time windows, there was also no compelling change in fixation preferences for NP1 in the 'NP2 accented' condition (middle panel) $\left(\beta_{\text {logodds }}=0.22,95 \% \mathrm{Crl}=[-0.08,0.53]\right)$, indicating that the absence of a pitch accent on NP1 is not used (as much) to infer the givenness of the corresponding referent.

Whether listeners make inference about the discourse status of NP1 based on the accent status of NP2 (backwards inference) can be probed by the comparison between the second and third time windows. Having heard an early accent on NP1, listeners use the absence of an accent on NP2 (left panel) as additional evidence for a contrastive referent for NP1. There is a compelling increase in looks to the NP1 target referent (from $37 \%$ to $55 \%$ ) $\left(\beta_{\text {logodds }}=-0.73,95 \% \mathrm{Crl}=[-0.97,-0.49]\right.$ ). Notably, in this condition, the absence of an accent on NP2 is decisive about the referent of NP1, because an unaccented NP2 occurs only in the 'NP1 accented' condition. Evidence for backwards inference in the other two conditions remains inconclusive (NP2 accented: $\beta_{\text {logodds }}=-0.26,95 \% \mathrm{Crl}=[-0.69,0.16]$; Both NPs accented: $\beta_{\text {logodds }}=$ $0.21,95 \% \mathrm{Crl}=[-0.20,0.68])$

\subsection{Looks ahead to the second referent}

Beyond the local interpretation of prenuclear accents (i.e. in inferring the discourse status of NP1), it is informative to look at whether listeners use the early intonational information to infer the discourse status of the upcoming NP2 (bottom row). Only the '2nd NP accented' condition (middle panel) shows a change in preferences. The early window exhibits smaller preferences for the target NP2 (33\%) than the NP1 window (44\%). The model estimates a compelling difference between these windows $\left(\beta_{\text {logodds }}=-0.44,95 \% \mathrm{Crl}=[-0.71,-0.14]\right)$. In other words, when listeners do not hear an early pitch accent on NP1, they anticipate a contrastive NP2.

Within the microcosm of the experiment, an early pitch accent was highly predictable of the givenness of NP2, and yet we find no evidence that listeners used that information to predict the referent of NP2 (NP1 accented: $\beta_{\text {logodds }}=0.02,95 \%$ $\mathrm{Crl}=[-0.21,0.26]$; Both NPs accented: $\beta_{\text {logodds }}=0.07,95 \% \mathrm{Crl}=[-0.20,0.38]$. This 
important finding indicates that listeners are not strictly rational comprehenders who greedily use all available evidence. We return to this point in the general discussion.

\section{Discussion}

This visual-world eye-tracking study aimed at understanding how listeners integrate acoustic evidence of intonational events occurring in different regions of an utterance to interpret a complex referential scene. In the absence of an interpretable referring expression (participants heard "thingy"), the presence (or absence) of certain intonational cues allowed listeners early on not only to infer the intended local referent of "thingy", but also to anticipate the referent of a downstream expression. The results provide evidence that listeners can locally interpret early accents as contrastive. Similar results from a German eye-tracking study of early accents marking contrastive topics are reported by Braun and Biezma (2019).

In the present study, we find no conclusive evidence that listeners interpret the absence of a prenuclear accent as a local cue to referential status. Yet we find evidence that when encountering an unaccented word later in the phrase, i.e. on NP2, listeners make backwards inferences about NP1. Specifically, having heard an accent on the first NP and then encountering no pitch accent on the second NP, listeners become more confident in associating a contrastive referent with the first NP. We find no evidence for backward inference in the other two conditions, suggesting that while the absence of an accent on the final argument licenses an inference about the referential status of an earlier expression, a late accent does not license a similar 'backwards' inference. This finding likely reflects listeners' experience with the English intonation system, where each utterance has at least one pitch accent.

Based on their experience with English, when hearing an early accent, listeners cannot make a prediction about the occurrence of another accent later in the phrase, because utterances may or may not have multiple accents. But the absence of a late accent does increase listeners' certainty about the interpretation of the already encountered early accent as marking contrastive focus. After hearing no accent early on, however, listeners know that there must be at least one accent downstream. This is exactly what we observe. Listeners' preference for a contrastive NP2 increases after encountering the earlier NP1 with no accent. This is also in line with recent findings by Roettger and Franke (2019) in which listeners use the absence of 
an early accent to make inferences about the discourse status of a referent downstream.

Coming back to the theoretical question of whether intonational processing is incremental or holistic, our findings support both assumptions to some extent. Evidence that intonational processing is incremental is seen in the fact that listeners immediately integrate information from an early accent to infer the discourse status of the local referent, as has been previously claimed (Dahan et al. 2002, Weber et al. 2006, Ito \& Speer 2006, Kurumada et al. 2014, Braun \& Biezma 2019). Note that listeners process early accents before knowing their status as prenuclear (followed by another accent), or nuclear, suggesting that this distinction is not relevant to intonation processing. Incremental processing is different in the absence of an early accent. Our results show that an unaccented NP1 is uninformative about the local referent (looks to the given referent are not boosted), but it does trigger strong predictions about the discourse status of an upcoming referent as contrastive.

There is also limited evidence that intonational processing is holistic, in the sense that there is backward inference. An early accent on NP1 triggers looks to the contrastive referent, which increase further when there is no downstream accent, i.e., when NP2 is unaccented. It appears that when processing the unaccented NP2, listeners have clear evidence for the greater relative prominence of the NP1. In English, this prominence asymmetry is a strong cue to a contrastive referent for the early accented NP. On the other hand, an accented NP2 does not similarly trigger backward inference, i.e., does not increase looks to the given referent for NP1, even though the NP2 accent is informative as a downstream cue to the referential status of NP1 in the microcosm of the experiment. ${ }^{2}$

To summarize, neither pure incremental processing ("now-or-never") nor purely holistic processing explain our data. Listeners use early cues, late cues, and their relative prominence to probabilistically guide reference resolution of a complex scene. Given that information transmission through speech is noisy, it is most efficient to evaluate evidence probabilistically, maintain uncertainty about inferences and use available evidence downstream to resolve this uncertainty. Indeed, human

\footnotetext{
2 The absence of a backward inference from NP2 may be due to a ceiling affect for looks to the given referent. In the microcosm of the experiment, an accented NP2 is always paired with a given referent for NP1. Participants show an initial bias to look at the given referent for NP1, which persists unchanged across the accented NP2 region.
} 
cognition is characterized by exactly this type of rational integration of information (e.g. Anderson 1991, Geisler 2011). Consistent with this view, research on speech processing shows that listeners evaluate acoustic cues to phoneme and word identity in light of downstream acoustic information (McMurray et al. 2009, Connine, Blasko, \& Hall, 1991; Brown-Schmidt \& Toscano, 2017), modulated by its expected utility (Bushong \& Jaeger 2019).

\section{Conclusion}

This paper investigated how listeners integrate pitch information on multiple referring expressions within an utterance to interpret referential expressions in an ambiguous scene. Evidence from a visual world eye tracking study suggest that listeners incrementally process pitch accents as soon as they appear in the signal, and use accent information to reduce uncertainty about the referents of local and downstream expressions. Listeners also process early and late pitch accents in relation to one another, such that early cues in the utterance can restrict later inferences and late cues can be used to resolve uncertainty associated with earlier cues. These findings have implications for a model of intonational processing, for which neither a local processing strategy nor a holistic view alone are sufficient. Effective comprehension of intonational events requires maintaining perceptual information long enough to integrate it with downstream intonational events. In speech comprehension, as in other domains of cognition, information processing is incremental and involves both forward and backward inference.

\section{References}

Allopenna, P. D., Magnuson, J. S., \& Tanenhaus, M. K. (1998). Tracking the time course of spoken word recognition using eye movements: Evidence for continuous mapping models. Journal of Memory and Language, 38(4), 419-439.

Anderson, J. R. (1990). The Adaptive Character of Thought. Lawrence Erlbaum. Bänziger, T., \& Scherer, K. R. (2005). The role of intonation in emotional expressions. Speech Communication, 46(3), 252-267. https://doi.org/10.1016/ j.specom.2005.02.016

Baumann, S., \& Schumacher, P. B. (2012). (De-)Accentuation and the Processing of Information Status: Evidence from Event-Related Brain Potentials. Language and Speech, 55(3), 361-381. https://doi.org/10.1177/0023830911422184 
Birch, S., \& Clifton, C. (1995). Focus, Accent, and Argument Structure: Effects on Language Comprehension. Language and Speech, 38(4), 365-391. https:// doi.org/10/gg466p

Bishop, J. (2017). Focus projection and prenuclear accents: Evidence from lexical processing. Language, Cognition and Neuroscience, 32(2), 236-253. https:// doi.org/10/gg466d

Boersma, P., \& Weenink, D. (2016). Praat: Doing phonetics by computer. [Computer program]. Version 6.0.17.

Bolinger, D. L. M. (1989). Intonation and its uses: Melody in grammar and discourse. Stanford University Press.

Braun, B., \& Biezma, M. (2019). Prenuclear L*+H Activates Alternatives for the Accented Word. Frontiers in Psychology, 10. https://doi.org/10/gg466q

Breen, M., Fedorenko, E., Wagner, M., \& Gibson, E. (2010). Acoustic correlates of information structure. Language and Cognitive Processes, 25(7-9), 1044-1098.

Brodeur, M. B., Dionne-Dostie, E., Montreuil, T., \& Lepage, M. (2010). The Bank of Standardized Stimuli (BOSS), a new set of 480 normative photos of objects to be used as visual stimuli in cognitive research. PloS One, 5(5), e10773.

Brown-Schmidt, S., \& Toscano, J. C. (2017). Gradient acoustic information induces long-lasting referential uncertainty in short discourses. Language, Cognition and Neuroscience, 32(10), 1211-1228. https://doi.org/ 10.1080/23273798.2017.1325508

Büring, D. (2007). Semantics, intonation and information structure. In G. Ramchand \& C. Reiss (Eds.), The Oxford handbook of linguistic interfaces (p. 445474). Oxford University Press. 10.1093/oxfordhb/9780199247455.013.0015

Bürkner, P.-C. (2016). brms: An R package for Bayesian multilevel models using Stan. Journal of Statistical Software, 80(1), 1-28.

Bushong, W., \& Jaeger, T. F. (2019). Modeling Long-Distance Cue Integration in Spoken Word Recognition. Proceedings of the Workshop on Cognitive Modeling and Computational Linguistics, 62-70. https://doi.org/10.18653/v1/W19-2907

Calhoun, S. (2010). The centrality of metrical structure in signaling information structure: A probabilistic perspective. Language, 86(1), 1-42. https://doi.org/10/cxrhdm

Christiansen, M. H., \& Chater, N. (2016). The Now-or-Never bottleneck: A fundamental constraint on language. Behavioral and Brain Sciences, 39, e62. https:// doi.org/10.1017/S0140525X1500031X

Cole, J. (2015). Prosody in context: A review. Language, Cognition and Neuroscience, 30(1-2), 1-31.

Cole, J., Hualde, J. I., Smith, C. L., Eager, C., Mahrt, T., \& Napoleão de Souza, R. (2019). Sound, structure and meaning: The bases of prominence ratings in Eng- 
lish, French and Spanish. Journal of Phonetics, 75, 113-147. https://doi.org/10/ gg466k

Connine, C. M., Blasko, D. G., \& Hall, M. (1991). Effects of subsequent sentence context in auditory word recognition: Temporal and linguistic constrainst. Journal of Memory and Language, 30(2), 234-250. https://doi.org/ 10.1016/0749-596X(91)90005-5

Cruttenden, A. (1997). Intonation. Cambridge University Press.

Cutler, A., Dahan, D., \& Van Donselaar, W. (1997). Prosody in the comprehension of spoken language: A literature review. Language and Speech, 40(2), 141-201.

Dahan, D. (2015). Prosody and language comprehension. Wiley Interdisciplinary Reviews: Cognitive Science, 6(5), 441-452.

Dahan, D., Tanenhaus, M. K., \& Chambers, C. G. (2002). Accent and reference resolution in spoken-language comprehension. Journal of Memory and Language, 47(2), 292-314.

Dennison, H. Y., \& Schafer, A. J. (2010). Online construction of implicature through contrastive prosody. Proceedings of the 5th International Conference on Speech Prosody.

Dennison, H. Y. (2010). Processing implied meaning through contrastive prosody. PhD dissertation, University of Hawaii, Manoa. http://www.ling.hawaii.edu/graduate/Dissertations/DennisonHeeYeonFinal.pdf

Geisler, W. S. (2011). Contributions of ideal observer theory to vision research. Vision Research, 51(7), 771-781. https://doi.org/10/czwfst

Gussenhoven, C. (1983). Focus, Mode and the Nucleus. Journal of Linguistics, 19(2), 377-417. https://doi.org/10/cz3hx3

Gussenhoven, C. (2004). The phonology of tone and intonation. Cambridge University Press.

Halliday, M. A. K. (1967). Intonation and grammar in British English. Mouton de Gruyter.

Heim, S., \& Alter, K. (2006). Prosodic pitch accents in language comprehension and production: ERP data and acoustic analyses. Acta Neurobiologiae Experimentalis, 66, 55-68.

Holliday, N. R. (2016). Intonational Variation, Linguistic Style and the Black/Biracial Experience. PhD dissertation, New York University

Ito, K., \& Speer, S. R. (2008). Anticipatory effects of intonation: Eye movements during instructed visual search. Journal of Memory and Language, 58(2), 541-573. Kapatsinski, V., Olejarczuk, P., \& Redford, M. A. (2017). Perceptual Learning of Intonation Contour Categories in Adults and 9- to 11-Year-Old Children: Adults Are 
More Narrow-Minded. Cognitive Science, 41(2), 383-415. https://doi.org/10/ f9w62c

Katz, J., \& Selkirk, E. (2011). Contrastive focus vs. discourse-new: Evidence from phonetic prominence in English. Language, 87(4), 771-816. https://doi.org/10/ gg5h56

Krahmer, E., \& Swerts, M. (2001). On the alleged existence of contrastive accents. Speech Communication, 34(4), 391-405. https://doi.org/10.1016/ S0167-6393(00)00058-3

Kurumada, C., Brown, M., Bibyk, S., Pontillo, D. F., \& Tanenhaus, M. K. (2014). Is it or isn't it: Listeners make rapid use of prosody to infer speaker meanings. Cognition, 133(2), 335-342.

Ladd, D. R. (2008). Intonational Phonology. Cambridge University Press.

McMurray, B., Tanenhaus, M. K., \& Aslin, R. N. (2009). Within-category VOT affects recovery from "lexical" garden-paths: Evidence against phoneme-level inhibition. Journal of Memory and Language, 60(1), 65-91. https://doi.org/10.1016/ j.jml.2008.07.002

Petrone, C., \& D'Imperio, M. (2011). From Tones to Tunes: Effects of the f0 Prenuclear Region in the Perception of Neapolitan Statements and Questions. In S. Frota, G. Elordieta, \& P. Prieto (Eds.), Prosodic Categories: Production, Perception and Comprehension (pp. 207-230). Springer Netherlands. https://doi.org/ 10.1007/978-94-007-0137-3 9

Petrone, C., \& Niebuhr, O. (2014). On the intonation of German intonation questions: The role of the prenuclear region. Language and Speech, 57(1), 108-146. https:// doi.org/10/f5t75v

Pierrehumbert, J., \& Hirschberg, J. (1990). The meaning of intonational contours in the interpretation of discourse. In P. Cohen, J. Morgan, \& M. Pollack (Eds.), Intentions in Communication (pp. 271-311). MIT Press.

R Core Team. (2019). R: A Language and Environment for Statistical Computing. $\mathrm{R}$ Foundation for Statistical Computing. https://www.R-project.org/

Roettger, T. B., \& Franke, M. (2019). Evidential Strength of Intonational Cues and Rational Adaptation to (Un-)Reliable Intonation. Cognitive Science, 43(7), e12745. https://doi.org/10.1111/cogs.12745

Swerts, M., \& Geluykens, R. (1994). Prosody as a marker of information flow in spoken discourse. Language and Speech, 21-43. https://doi.org/10/gg5h55

Weber, A., Braun, B., \& Crocker, M. W. (2006). Finding referents in time: Eye-tracking evidence for the role of contrastive accents. Language and Speech, 49(3), 367-392. 